\title{
General Vertex-Distinguishing Total Coloring of Graphs
}

\author{
Chanjuan Liu and Enqiang Zhu \\ School of Electronics Engineering and Computer Science, Peking University, Beijing 100871, China \\ Correspondence should be addressed to Enqiang Zhu; zhuenqiang@163.com
}

Received 24 April 2014; Accepted 14 July 2014; Published 3 August 2014

Academic Editor: Tak-Wah Lam

Copyright (C) 2014 C. Liu and E. Zhu. This is an open access article distributed under the Creative Commons Attribution License, which permits unrestricted use, distribution, and reproduction in any medium, provided the original work is properly cited.

\begin{abstract}
The general vertex-distinguishing total chromatic number of a graph $G$ is the minimum integer $k$, for which the vertices and edges of $G$ are colored using $k$ colors such that any two vertices have distinct sets of colors of them and their incident edges. In this paper, we figure out the exact value of this chromatic number of some special graphs and propose a conjecture on the upper bound of this chromatic number.
\end{abstract}

\section{Introduction}

All graphs considered in this paper are simple and finite. For a graph $G$, we denote by $V(G), E(G), \Delta(G)$, and $\delta(G)$ the sets of vertices, edges, maximum degree, and minimum degree of $G$, respectively. For a vertex $v$ of $G, d_{G}(v)$ is the degree of $v$ in $G$. For any $V^{\prime} \subseteq V(G)$, we use $G\left[V^{\prime}\right]$ to denote the subgraph induced by $V^{\prime}$. For any undefined terms, the reader is referred to the book [1].

The coloring problem of graphs is one of the classical research areas in graph theory. It has been widely applied to various fields, such as large scheduling [2], assignment of radio frequency [3], and separating combustible chemical combinations [4]. Due to its extensive application, many new variants of colorings have been studied [5].

Recall that a $k$-edge coloring of a graph $G$ is a mapping $f: E(G) \rightarrow C$, where $C$ is a set of $k$ colors. An edge coloring is proper if adjacent edges receive distinct colors. In 1985, Harary and Plantholt [6] first considered pointdistinguishing chromatic index, which is a variant of edge coloring. After that, many other variants of edge coloring were introduced, such as vertex-distinguishing proper edge coloring [7], adjacent vertex-distinguishing edge coloring [8], and general adjacent vertex-distinguishing edge coloring [9].

A total $k$-coloring of a graph $G$ is a coloring of $V(G) \cup E(G)$ using $k$ colors. A total $k$-coloring is proper if no two adjacent or incident elements receive the same color. The minimum number of colors required for a proper total coloring of $G$ is called the total chromatic number of $G$ and is denoted by $\chi_{t}(G)$. Behzad [10] and Vizing [11] independently made the conjecture that, for any graph $G$,

$$
\chi_{t}(G) \leq(G)+2 .
$$

This is known as the total coloring conjecture (TCC) and is still unproven.

Let $f$ be a total $k$-coloring of $G$. The total color set (with respect to $f$ ) of a vertex $v \in V(G)$ is the set, denoted by $C_{f}(v)$, of colors of $v$ and its incident edges. We denote by $\mathscr{C}_{f}(G)$ the set of total color sets of all vertices of $G$. Furthermore, let $S$ be a subset of $V(G) \cup E(G)$; we use $C_{f}(S)$ to denote the set of colors of elements of $S$.

Like edge coloring, total coloring also has some variants. In 2005, Zhang et al. [12] added a restriction to the definition of total coloring and proposed a new type of coloring defined as follows.

Definition 1. Let $f$ be a proper total $k$-coloring of a graph $G$. If, for all $u, v \in V(G), C_{f}(u) \neq C_{f}(v)$, then $f$ is called an adjacent vertex-distinguishing total $k$-coloring of $G$, or a $k$-AVDTC of $G$ for short. The minimum number $k$ for which $G$ has a $k$-AVDTC is the adjacent vertex-distinguishing total chromatic number of $G$, denoted by $\chi_{a t}(G)$.

Zhang et al. [12] conjectured that, for any graph $G$, it has

$$
\chi_{a t}(G) \leq \Delta(G)+3 .
$$


In [13-15], authors proved that there exists a 6-AVDTC of graphs with $\Delta=3$, which indicates conjecture (2) holds for such graphs. For further research on adjacent vertexdistinguishing total chromatic number, one may refer to [1623].

For a $k$-AVDTC $f$ of a graph $G$, if $C_{f}(u) \neq C_{f}(v)$ is required for any two distinct vertices $u, v$, then $f$ is called a vertex-distinguishing total $k$-coloring of $G$, abbreviated as $k$-VDTC. The minimum number $k$ such that $G$ has a $k$-VDTC is called the vertex-distinguishing total chromatic number, denoted by $\chi_{v t}(G)[24]$. Zhang et al. conjectured in [24] that, for any graph $G$, it follows that

$$
\mu_{t}(G) \leq \chi_{v t}(G) \leq \mu_{t}(G)+1
$$

where $\mu_{t}(G)=\min \left\{k \mid\left(\begin{array}{c}k \\ i+1\end{array}\right) \geq n_{i}, \delta \leq i \leq \Delta\right\}$.

In this paper, we introduce a variant of vertexdistinguishing total coloring of a graph $G$, which relaxes the restriction that the coloring is proper. We now present the detailed definition as follows.

Definition 2. Let $G$ be a graph and $k$ be a positive integer. A total coloring $f$ of $G$ using $k$ colors is called a general vertex-distinguishing total $k$-coloring of $G$ (or $k$-GVDTC of $G$ briefly) if, for all $u, v \in V(G), C_{f}(u) \neq C_{f}(v)$. The minimum number $k$ for which $G$ has a $k$-GVDTC is the general vertexdistinguishing total chromatic number, denoted by $\chi_{g v t}(G)$.

It is evident that $\chi_{g v t}(G)$ does exist for any graph $G$. In this paper, we study the general vertex-distinguishing total coloring of some special classes of graphs and obtain the exact value of the general vertex-distinguishing total chromatic number of these graphs. Furthermore, we propose a conjecture on the upper bound of general vertex-distinguishing total chromatic number of a graph.

\section{Main Results}

We first present a trivial lower bound on the general vertexdistinguishing total chromatic number of a graph.

Theorem 3. Let $G$ be a graph on $n$ vertices. Then

$$
\chi_{\text {gvt }}(G) \geq\left\lceil\log _{2}(n+1)\right\rceil .
$$

Proof. Let $\chi_{g v t}(G)=k$. It follows that $n \leq\left(\begin{array}{c}k \\ 1\end{array}\right)+\left(\begin{array}{c}k \\ 2\end{array}\right)+\cdots+$ $\left(\begin{array}{l}k \\ k\end{array}\right)=2^{k}-1$, so $k \geq\left\lceil\log _{2}(n+1)\right\rceil$.

Notice that the lower bound of Theorem 3 can be attained in graphs, such as the $n$-vertex path $P_{n}$ for $n=1,2, \ldots, 7$. One can readily check that $\chi_{\text {gvt }\left(P_{1}\right)}=1$ and $\chi_{g v t\left(P_{n}\right)}=2$ for $n=2,3$ and $\chi_{g v t\left(P_{n}\right)}=3$ for $n=4,5,6,7$.

Theorem 4. Let $G$ be a graph without isolated vertices and isolated edges. Then

$$
\chi_{g v t}(G) \leq \chi_{g v d}^{\prime}(G) .
$$

Proof. Suppose that $f$ is a $k$-GVDEC of $G$. For any $u \in V(G)$, let $f(u)=f(u v)$, where $u v \in E(G)$. Obviously, $f$ is a $k$-GVDTC of $G$.
We now turn to investigating the general vertexdistinguishing total chromatic number of an $n$-vertex path.

Theorem 5. Let $P_{n}$ be a path on $n$ vertices, $n \geq 1$. Then

$$
\chi_{g v t}\left(P_{n}\right)=\left\lceil\sqrt[3]{3 n+\sqrt{9 n^{2}+\frac{125}{27}}}+\sqrt[3]{3 n-\sqrt{9 n^{2}+\frac{125}{27}}}\right\rceil .
$$

Proof. Denote by $P_{n}=v_{1} v_{2} \cdots v_{n}$ a path $P_{n}$ with vertex set $\left\{v_{1}, v_{2}, \ldots, v_{n}\right\}$ and edge set $\left\{v_{1} v_{2}, v_{2} v_{3}, \ldots, v_{n-1} v_{n}\right\}$. Let $\chi_{\text {gvt }}\left(P_{n}\right)=k$, and let $f$ be a $k$-GVDTC of $P_{n}$. Let $\alpha_{k}=\left(\begin{array}{c}k-1 \\ 1\end{array}\right)+$ $\left(\begin{array}{c}k-1 \\ 2\end{array}\right)+\left(\begin{array}{c}k-1 \\ 3\end{array}\right), \beta_{k}=\left(\begin{array}{l}k \\ 1\end{array}\right)+\left(\begin{array}{l}k \\ 2\end{array}\right)+\left(\begin{array}{l}k \\ 3\end{array}\right), \gamma_{k}=1+\left(\begin{array}{c}k-1 \\ 1\end{array}\right)+\left(\begin{array}{c}k-1 \\ 2\end{array}\right)$, and

$$
k^{*}=\left\lceil\sqrt[3]{3 n+\sqrt{9 n^{2}+\frac{125}{27}}}+\sqrt[3]{3 n-\sqrt{9 n^{2}+\frac{125}{27}}}\right\rceil .
$$

Evidently, $\left|C_{f}\left(v_{i}\right)\right| \leq 3$, and $n \leq \beta_{k}$ (which implies $k \geq$ $\left.k^{*}\right)$. In order to prove the conclusion, $k=k^{*}$, it suffices to give a $k^{*}$-GVDTC of $P_{n}$. When $n \leq 7$, it is not hard to construct the corresponding general vertex-distinguishing total colorings. Let $n \geq 8$. We first construct a $k^{*}$-GVDTC $f^{\prime}$ of $P_{\beta_{k}}$ recursively. Note that when $n=\beta_{k}$, it has $k^{*}=k$.

Procedure 1. Construct a 4-GVDTC $f_{4}$ of $P_{\beta_{4}}$ (i.e., $P_{14}$ ) as follows: the vertices $v_{1}, v_{2}, \ldots, v_{14}$ are colored by $1,3,4,2,3,1$, $4,2,3,4,3,4,4,1$, respectively; the edges $v_{1} v_{2}, v_{2} v_{3}$, $v_{3} v_{4}, \ldots, v_{13} v_{14}$ are colored by $1,3,1,1,2,4,2,2,2,3,3,4,4$, respectively. It is easy to see that $f_{4}$ is a $4-G V D T C$ of $P_{14}$.

Procedure 2. Construct a $k$-GVDTC $f_{k}$ of $P_{\beta_{k}}$ based on a $(k-$ 1)-GVDTC $f_{k-1}$ of $P_{\beta_{k-1}}$. Let $f_{k}$ be

$$
\begin{gathered}
f_{k}\left(v_{i+\gamma_{k}}\right)=f_{k-1}\left(v_{i}\right)+1, \quad i=1,2, \ldots, \alpha_{k}-1 ; \\
f_{k}\left(v_{\alpha_{k}+\gamma_{k}}\right)=f_{k-1}\left(v_{\alpha_{k}}\right)=1 ; \\
f_{k}\left(v_{i+\gamma_{k}} v_{i+1+\gamma_{k}}\right)=f_{k-1}\left(v_{i} v_{i+1}\right)+1, \quad i=1,2, \ldots, \alpha_{k}-1 ; \\
f_{k}\left(v_{1}\right)=1, \quad f_{k}\left(v_{\gamma_{k}}\right)=k ;
\end{gathered}
$$

$v_{2}, v_{3}, \ldots, v_{\gamma_{k}-1}$ are colored by

$$
\underbrace{k-1, k}_{2 \text { elements }}, \underbrace{k-2, k-1, k}_{3 \text { elements }}, \ldots, \underbrace{2,3, \ldots, k}_{k-1 \text { elements }}
$$

when $k$ is even, $v_{1} v_{2}, v_{2} v_{3}, v_{3} v_{4}, \ldots, v_{\gamma_{k}} v_{\gamma_{k+1}}$ are colored by 


$$
\begin{aligned}
& \underbrace{1}_{1 \text { element }}, \underbrace{k-1,1}_{2 \text { elements }}, \underbrace{1, k-2,1}_{3 \text { elements }}, \underbrace{k-3,1, k-3,1}_{4 \text { elements }}, \underbrace{1, k-4,1, k-4,1}_{5 \text { elements }}, \\
& \vdots \\
& \underbrace{1, k-(k-4), \ldots, 1, k-(k-4), 1}_{k-3 \text { elements }}, \underbrace{k-(k-3), 1, \ldots, k-(k-3), 1}_{k-2 \text { elements }}, \\
& \underbrace{2,2}_{k-2,1, \ldots, 2,1} \text {; } \\
& \text { k-2 elements } 2 \text { elements }
\end{aligned}
$$

when $k$ is odd, $v_{1} v_{2}, v_{2} v_{3}, v_{3} v_{4}, \ldots, v_{\gamma_{k}} v_{\gamma_{k+1}}$ are colored by

$$
\begin{aligned}
& \underbrace{1}_{1 \text { element }}, \frac{k-1,1}{2 \text { elements }}, \underbrace{1, k-2,1}_{3 \text { elements }}, \underbrace{k-3,1, k-3,1}_{4 \text { elements }}, \underbrace{1, k-4,1, k-4,1}_{5 \text { elements }}, \\
& \vdots \\
& \underbrace{k-(k-4), 1, \ldots, k-(k-4), 1}_{k-3 \text { elements }}, \underbrace{1, k-(k-3), \ldots, 1, k-(k-3), 1}_{k-2 \text { elements }}, \\
& \underbrace{1,2, \ldots, 1,2,1}_{k-2 \text { elements }}, \underbrace{2,2}_{2 \text { elements }} \text {. }
\end{aligned}
$$

It should be pointed out that when $j$ is odd for $j \in$ $\{1,2, \ldots, k-3\}$, the colors' form is $k-j, 1, k-j, 1, \ldots, k-j, 1$ with totally $j+1$ elements, and when $j$ is even for $j \in$ $\{1,2, \ldots, k-3\}$, the colors' form is $1, k-j, 1, k-j, \ldots, 1, k-j, 1$ with $j+1$ elements in total.

According to $f_{k-1}$, we can see that, for any $i, j=\gamma_{k}+$ $1, \gamma_{k}+2, \ldots, \beta_{k}-1$, and $i \neq j$, it follows that $\{2, k\}$ is not a total color set of vertices $v_{i}, 1 \notin C_{f_{k}}\left(v_{i}\right)$, and $C_{f_{k}}\left(v_{i}\right) \neq$ $C_{f_{k}}\left(v_{j}\right)$. In addition, $C_{1}, C_{2}, \ldots, C_{\gamma-1}$ are as follows: $\{1\}(1$ item), $\{\{1, k-1\},\{1, k-1, k\}\}$ (2 items), $\{\{1, k-2\},\{1, k-$ $2, k-1\},\{1, k-2, k\}\}$ (3 items), .., \{\{1,k-j\},\{1,k-j,k$j+1\},\{1, k-j, k-j+2\}, \ldots,\{1, k-j, k\}\}(j+1$ items), $\ldots$, and $\{\{1,2\},\{1,2,3\},\{1,2,4\}, \ldots,\{1,2, k\}\}$ ( $k-1$ items). And $C_{f_{k}}\left(v_{\gamma_{k}}\right)=\{2, k\}$. So, $f_{k}$ is a $k$-GVDTC of $P_{\beta_{k}}$. We now show that $P_{n}$ also has a $k$-GVDTC based on a $k$-GVDTC of $P_{\beta_{k}}$, for $\left(\begin{array}{c}k-1 \\ 1\end{array}\right)+\left(\begin{array}{c}k-1 \\ 2\end{array}\right)+\left(\begin{array}{c}k-1 \\ 3\end{array}\right)<n<\beta_{k}$.

Let $r=\beta-n$, and let $f$ be a $k$-GVDTC of $P_{\beta_{k}}$ constructed by Procedures 1 and 2. We first delete $r$ vertices $v_{1}, v_{2}, \ldots, v_{r}$ from $P_{\beta_{k}}$. Obviously, the resulting graph, denoted by $v_{r+1} v_{r+2} \cdots v_{\beta_{k}}$, is isomorphic to $P_{n}$. Let $f^{\prime}$ be $f^{\prime}\left(v_{i} v_{i+1}\right)=f\left(v_{i} v_{i+1}\right)$ for $i=r+1, r+2, \ldots, \beta_{k}-1 ; f^{\prime}\left(v_{i}\right)=$ $f\left(v_{i}\right)$ for $i=r+2, \ldots, \beta$; and $f^{\prime}\left(v_{r+1}\right)=1$. Then $f^{\prime}$ is a $k$ GVDTC of $P_{n}$.

All the above show that the conclusion holds.

According to Theorem 5, we have the same conclusion on cycles. Let $C_{n}=v_{1} v_{2} \cdots v_{n} v_{1}$ be an $n$-vertex cycle with vertex set $\left\{v_{1}, v_{2}, \ldots, v_{n}\right\}$ and edge set $\left\{v_{1} v_{2}, v_{2} v_{3}, \ldots, v_{n-1} v_{n}, v_{n} v_{1}\right\}$.
Corollary 6. For any cycle $C_{n}(n \geq 3)$, one has

$$
\chi_{\text {gvt }}\left(C_{n}\right)=\left\lceil\sqrt[3]{3 n+\sqrt{9 n^{2}+\frac{125}{27}}}+\sqrt[3]{3 n-\sqrt{9 n^{2}+\frac{125}{27}}}\right\rceil .
$$

Proof. Let $C_{n}=v_{1} v_{2} \cdots v_{n} v_{1}$ and $P_{n}=C_{n} \backslash v_{1} v_{n}$; let also

$$
k^{*}=\left\lceil\sqrt[3]{3 n+\sqrt{9 n^{2}+\frac{125}{27}}}+\sqrt[3]{3 n-\sqrt{9 n^{2}+\frac{125}{27}}}\right\rceil
$$

and let $f$ be a $k^{*}$-GVETC of $P_{n}$, constructed by the method of Theorem 5. Then we can extend $f$ to a $k^{*}$-GVETC of $C_{n}$ by assigning color 1 to edge $v_{1} v_{n}$. So, the conclusion holds.

In the following (Theorem 7 to Theorem 9), we discuss the general vertex-distinguishing total chromatic number of some kinds of special trees. A star $S_{n}$ is the complete bipartite graph $K_{1, n}(n \geq 1)$. A double star $S_{m, n}$ is a tree containing exactly two vertices that are not leaves (which are necessarily adjacent). A tristar $S_{p, q, r}$ is a tree with vertex set $V\left(S_{p, q, r}\right)=$ $\left\{u_{i} \mid i=0,1, \ldots, p\right\} \cup\left\{v_{i} \mid i=0,1, \ldots, q\right\} \cup\left\{w_{i} \mid i=0,1, \ldots, \ell\right\}$ and edge set $E\left(S_{p, q, r}\right)=\left\{u_{0} u_{i} \mid i=1,2, \ldots, p\right\} \cup\left\{v_{0} v_{i} \mid i=\right.$ $1,2, \ldots, q\} \cup\left\{w_{0} w_{i} \mid i=1,2, \ldots, r\right\} \cup\left\{u_{0} v_{0}, v_{0} w_{0}\right\}$, where $p, q, r$ are positive integers.

Theorem 7. For a star $S_{n}(n \geq 1)$, one has

$$
\chi_{\text {gvt }}\left(S_{n}\right)= \begin{cases}2, & n=1,2 ; \\ \left\lceil\frac{\sqrt{8 n+1}-1}{2}\right\rceil, & n \geq 3 .\end{cases}
$$


Proof. When $n=1,2$, the conclusion is trivial. For $n \geq 3$, let $\chi_{\text {gvt }}\left(S_{n}\right)=k$, and $\lceil(\sqrt{8 n+1}-1) / 2\rceil=k^{\prime}$. Since, for any $u \in V\left(S_{n}\right) \backslash u_{0}\left(u_{0}\right.$ is the vertex with $\left.d_{S_{n}}\left(u_{0}\right) \geq 2\right),\left|C_{f}(u)\right| \leq 2$ for any $k$-GVDTC $f$ of $S_{n}$, it follows that $n \leq\left(\begin{array}{c}k \\ 1\end{array}\right)+\left(\begin{array}{c}k \\ 2\end{array}\right)$; that is, $k \geq k^{\prime}$. In order to prove $k=k^{\prime}$, we need to show that there exists a $k^{\prime}$-GVDTC of $S_{n}$. Otherwise, let $S_{n^{*}}$ be the graph with minimum $n^{*}$ such that $S_{n^{*}}$ does not have a $k^{\prime}$-GVDTC, where $n^{*} \leq n$. Let $u$ be a vertex of degree 1 in $S_{n^{*}}$. Consider the graph $G^{\prime}=S_{n^{*}}-u$, obtained from $S_{n^{*}}$ by deleting the vertex $u$ and its incident edge. By the assumption of $S_{n^{*}}, G^{\prime}$ has a $k^{\prime}$-GVDTC, denoted by $f^{\prime}$. In addition, by interchanging the colors of some vertex and its incident edge appropriately, we can assume $\left|C_{f}^{\prime}\left(u_{0}\right)\right| \geq 2$. Since $n^{*}-1<\left(\begin{array}{c}k^{\prime} \\ 1\end{array}\right)+\left(\begin{array}{c}k^{\prime} \\ 2\end{array}\right)$, there is at least one set $\{a, b\}$, for $a, b \in\left\{1,2, \ldots, k^{\prime}\right\}$, which is not the total color set of the vertices of $G^{\prime}$. So, on the basis of $f^{\prime}$, in $S_{n^{*}}$ we can color $u$ and its incident edge $u u_{0}$ by $a$ and $b$, respectively. Obviously, the resulting coloring is a $k^{\prime}$-GVDTC of $S_{n^{*}}$.

For two vertices $u, v$ of a graph $G$, to identify these two vertices is to replace them by a single vertex (denoted by $u-v$ in this paper) incident to all the edges which were incident in $G$ to either $u$ or $v$. The resulting graph is denoted by $G /\{u, v\}$. In what follows, we denoted by $[1, k]$ the set of $\{1,2, \ldots, k\}$.

Theorem 8. Let $S_{m, n}(m \geq n \geq 1)$ be a double star, and $\ell=$ $m+n$. Then

$$
\chi_{g v t}\left(S_{m, n}\right)= \begin{cases}3, & \ell=2,3,4,5 ; \\ 4, & \ell=6 \\ \left\lceil\frac{\sqrt{8 \ell+1}-1}{2}\right\rceil, & \ell \geq 7 .\end{cases}
$$

Proof. When $\ell \leq 6$, the results are easy to be proved. When $\ell \geq 7$, let $u, v$ be two vertices with degree more than 1 , and $G^{\prime}=S_{m, n} /\{u, v\}$. Evidently, the graph $G^{\prime}$ is isomorphic to the star $S_{\ell}$. Let $k^{\prime}=\lceil(\sqrt{8 \ell+1}-1) / 2\rceil$. Since $\left|C_{f}(x)\right| \leq 2$ for any $x \in V\left(S_{m, n}\right) \backslash\{u, v\}$, we have $\chi_{g v t}\left(S_{m, n}\right) \geq k^{\prime}$.

By Theorem $7, G^{\prime}$ contains a $k^{\prime}$-GVDTC $f^{\prime}$. Evidently, $\left|C_{f^{\prime}}(x)\right| \leq 2$ for any $x \in V\left(G^{\prime}\right) \backslash\{u-v\}$ and $\left|C_{f^{\prime}}(u-v)\right| \leq k^{\prime}$. If $\left|C_{f^{\prime}}(u-v)\right| \leq 2$, then we can extend $f^{\prime}$ to a $k^{\prime}$-GVDTC of $S_{m, n}$ by coloring vertices $u, v$ and edge $u v$ with any three different colors in $[1, k] \backslash\left\{f^{\prime}(u-v)\right\}$; if $\left|C_{f^{\prime}}(u-v)\right|=\ell^{\prime} \geq 3$, we without loss of generality assume $C_{f^{\prime}}(u-v) \backslash f^{\prime}(u-v)=\left[1, \ell^{\prime}\right]$. Let $E_{u}$ (resp., $E_{v}$ ) be the set of edges (except edge $u v$ ) incident to $u$ (resp., $v$ ) in $S_{m, n}$. We now extend $f^{\prime}$ to a $k^{\prime}$-GVDTC of $S_{m, n}$ as follows. By the fact that there remain vertices $u, v$ and edge $u v$ uncolored in $S_{m, n}$ when $f^{\prime}$ is restricted to $S_{m, n}$, we consider the following two cases. First, one of $u, v$, say $u$, satisfies that $C_{f^{\prime}}\left(E_{u}\right)$ contains at most two elements. Assume $C_{f^{\prime}}\left(E_{u}\right) \subseteq\{1,2\}$; we then color $u, u v, v$ by $2,3, c$, respectively, where $c=4$ when $C_{f^{\prime}}\left(E_{u}\right) \neq\{3,4\}$ and $c=2$ when $C_{f^{\prime}}\left(E_{u}\right)=\{3,4\}$. The resulting coloring of $S_{m, n}$ is also denoted by $f^{\prime}$. Then it follows in $S_{m, n}$ that $\left|C_{f^{\prime}}(u)\right| \geq 3,\left|C_{f^{\prime}}(v)\right| \geq 3$, and $4 \notin C_{f^{\prime}}(u)$ and $4 \in C_{f^{\prime}}(v)$. So, $f^{\prime}$ is a $k^{\prime}$-GVDTC of $S_{m, n}$. Second, $\left|C_{f^{\prime}}\left(E_{v}\right)\right| \geq 3$ and $\left|C_{f^{\prime}}\left(E_{u}\right)\right| \geq 3$; then we will further discuss two subcases.
(1) Consider $\left|C_{f}^{\prime}\left(E_{u}\right)\right|=\left|C_{f}^{\prime}\left(E_{v}\right)\right|=k^{\prime}$. Suppose that $V_{u}$ (and $V_{v}$ ) is the set of vertices, except $v$ (or $u$ ), adjacent to $u$ (and $v$ ) in $S_{m, n}$. Because $f^{\prime}$ is a $k^{\prime}$-GVDTC of $G^{\prime}$, either $V_{u}$ or $V_{v}$ contains no vertices with total color set $\{i\}$, for some $i \in\left\{1,2, \ldots, k^{\prime}\right\}$. Without loss of generality we assume that there is no vertex $x \in$ $V_{u}$ with $C_{f}^{\prime}(x)=\{i\}$. For any vertex $y$ in $V_{u}$ such that $C_{f}^{\prime}(y)=\{i, j\}$ and $f^{\prime}(y u)=i$, interchange the two colors of $y$ and $y u$. The resulting coloring, still denoted by $f^{\prime}$, satisfies that $C_{f^{\prime}}\left(E_{u}\right)$ does not contain color $i$. Then we color $u, u v, v$ by any three colors in $\left\{1,2, \ldots, k^{\prime}\right\} \backslash\{i\}$ and obtain a $k^{\prime}$-GVDTC of $S_{m, n}$.

(2) Consider $\left|C_{f}^{\prime}\left(E_{u}\right)\right|<k^{\prime}$ or $\left|C_{f}^{\prime}\left(E_{v}\right)\right|<k^{\prime}$; assume $\left|C_{f}^{\prime}\left(E_{u}\right)\right|<k^{\prime}$ here. Let $i \notin C_{f}^{\prime}\left(E_{u}\right)$. Color $v$ by $i$ and color $u, u v$ by any two colors in $\left[1, k^{\prime}\right] \backslash\{i\}$. Obviously, the resulting coloring is a $k^{\prime}-\mathrm{GVDTC}$ of $S_{m, n}$. holds.

All the above show that $\chi_{g v t}\left(S_{m, n}\right) \leq k^{\prime}$. So, the conclusion

Theorem 9. Let $S_{p, q, \ell}$ be a tristar defined as above, and $\ell=$ $p+q+r$. Then

$$
\chi_{g v t}\left(S_{p, q, r}\right)= \begin{cases}3, & \ell=3,4 \\ 4, & \ell=5,6 \\ \lceil(\sqrt{8 r+1}-1) / 2\rceil, & \ell \geq 7\end{cases}
$$

Proof. When $\ell=3,4$, the conclusion is easy to be checked; when $\ell=5$ or 6 , since $\left|V\left(S_{p, q, r}\right)\right| \geq 8$ and $\left(\begin{array}{l}3 \\ 1\end{array}\right)+\left(\begin{array}{l}3 \\ 2\end{array}\right)+\left(\begin{array}{l}3 \\ 3\end{array}\right)=$ 7 , it follows that $\chi_{g v t}\left(S_{p, q, r}\right)>3$. In addition, it is not hard to give a 4-GVDTC of $V\left(S_{p, q, r}\right)$ in each case of $\ell=5$ or 6 , so $\chi_{g v t}\left(S_{p, q, r}\right)=4$; when $\ell \geq 7$, let $k^{\prime}=\lceil(\sqrt{8 r+1}-1) / 2\rceil$. Identify vertices $u_{0}$ and $v_{0}$ in $S_{p, q, r}$ and let $G^{\prime}=S_{p, q, r} /\{u, v\}$. By Theorem $8, G^{\prime}$ has a $k^{\prime}$-GVDTC $f^{\prime}$. With the analogous analysis method of Theorem 8 , we can also extend $f^{\prime}$ to a $k^{\prime}$ GVDTC of $S_{p, q, r}$. This shows $\chi_{g v t}\left(S_{p, q, r}\right) \leq k^{\prime}$. On the other hand, for any $k$-GVDTC $f$ of $S_{p, q, r}$, it has that $\ell \leq\left(\begin{array}{c}k \\ 1\end{array}\right)+\left(\begin{array}{c}k \\ 2\end{array}\right)$; that is, $k \geq k^{\prime}$. So, the result holds.

In the above, we construct a $k$-GVDTC of a graph $G$ by extending a $k$-GVDTC of graph $G^{\prime}$, where $G^{\prime}$ is the resulting graph of identifying two vertices of degree more than 1 in $G$. But this method does not always work. For instance, the graph $G /\{u, v\}$ shown in Figure 1(b) has a 4-GVDTC, but the graph $G$ shown in Figure 1(a) does not contain any 4-GVDTC. So any 4-GVDTC of $G /\{u, v\}$ can not be extended to a $4-G V D T C$ of $G$.

In the following we are devoted to the study of the general vertex-distinguishing chromatic number of fan graph $F_{n}$, wheel graph $W_{n}$, and complete graph $K_{n}$. Let $G, H$ be two graphs such that $V(G) \cap V(H)=\emptyset$. The join $G+H$ of $G$ and $H$ is a graph with vertex set $V(G+H)=V(G) \cup V(H)$ and edge set $E(G+H)=E(G) \cup E(H) \cup\{u v \mid u \in V(G), v \in V(H)\}$. A fan graph $F_{n}$ is defined as the join of a path of $n$ vertices and an isolated vertex. A wheel graph $W_{n}$ is defined as the join of a cycle of $n$ vertices and an isolated vertex. 


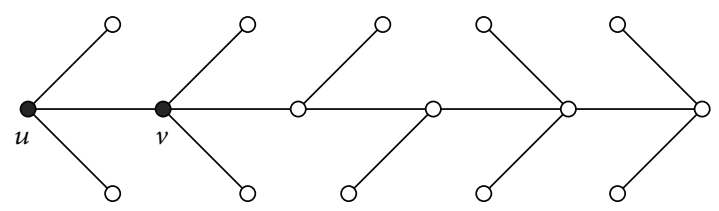

(a)

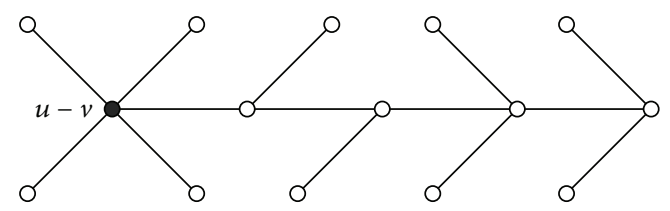

(b)

Figure 1: (a) A graph, $G$; (b) $G /\{u, v\}$.

Theorem 10. Let $F_{n}$ be a fan, $n \geq 2$; then

$$
\chi_{g v t}\left(F_{n}\right)= \begin{cases}3, & n=2,3, \ldots, 6 \\ 4, & n=7,8, \ldots, 14 \\ k, & n \geq 15\end{cases}
$$

where $\left(\begin{array}{c}k-1 \\ 1\end{array}\right)+\left(\begin{array}{c}k-1 \\ 2\end{array}\right)+\left(\begin{array}{c}k-1 \\ 3\end{array}\right)+\left(\begin{array}{c}k-1 \\ 4\end{array}\right)<n \leq\left(\begin{array}{l}k \\ 1\end{array}\right)+\left(\begin{array}{l}k \\ 2\end{array}\right)+\left(\begin{array}{l}k \\ 3\end{array}\right)+\left(\begin{array}{l}k \\ 4\end{array}\right)$.

Proof. Let $V\left(F_{n}\right)=\left\{v_{i} \mid i=0,1, \ldots, n\right\}$ and $E\left(F_{n}\right)=$ $\left\{v_{1} v_{2}, v_{2} v_{3}, \ldots, v_{n-1} v_{n}\right\} \cup\left\{v_{0} v_{i} \mid i=1,2, \ldots, n\right\}$. When $n \leq 14$, the conclusion is easy to show. We now consider the case of $n \geq 15$ (which implies $k \geq 5)$. Since $\left(\begin{array}{c}k-1 \\ 1\end{array}\right)+\left(\begin{array}{c}k-1 \\ 2\end{array}\right)+\left(\begin{array}{c}k-1 \\ 3\end{array}\right)+$ $\left(\begin{array}{c}k-1 \\ 4\end{array}\right)<n$ and $\left|C_{f}\left(v_{i}\right)\right| \leq 4$ for $i \in[1, n]$, we can easily deduce that $\chi_{\text {gvt }}\left(F_{n}\right) \geq k$. So, it suffices to show that $F_{n}$ contains a $k$ GVDTC. In particular, we prove that $F_{n}$ contains a $k$-GVDTC such that the total color set of $v_{0}$ contains at least 5 elements. By induction on $n$. When $n=15$, it is not hard to construct such a $k(\geq 5)$-GVDTC of $F_{n}$. Suppose that, for any $F_{n^{\prime}}, n^{\prime}<n$, there exists a $k$-GVDTC of $F_{n^{\prime}}$. Consider the fan graph $F_{n-1}$, and let $f$ be a $k$-GVDTC of $F_{n-1}$. Anyway, we can assume that, for any color $x \in[1, k]$, there is an edge $v_{i} v_{i+1}$ for some $i \in[1, n-1]$ such that $f\left(v_{i} v_{i+1}\right)=x$ (If not, we can permutate $x$ and $\left.f\left(v_{i} v_{i+1}\right)\right)$.

Note that for any edge $v_{i} v_{i+1}$ of $F_{n-1}, i \in[1, k]$, if we replace this edge by a vertex $u$ and connect this vertex to $v_{0}$, $v_{i}$, and $v_{i+1}$, then the resulting graph, denoted by $F_{n-1}^{v_{i}, u_{i+1}, v_{i+1}}$, is isomorphic to $F_{n}$. We will use this to construct a $k$-GVDTC of $F_{n}$ based on $f$. It is obvious that there remain only 4 uncolored elements, $v_{i} u, u v_{i+1}, u v_{0}$, and $u$ in $F_{n-1}^{v_{i}, u, v_{i+1}}$, if we restrict $f$ to $F_{n-1}^{v_{i}, u, v_{i+1}}$. We need to consider the following 2 cases.

Case 1. There exist colors $x, y, z \in[1, k]$ such that $\{x, y, z\} \notin$ $\mathscr{C}_{f}\left(F_{n-1}\right)$. Let $v_{i} v_{i+1}$ be the edge with $f\left(v_{i} v_{i+1}\right)=x, i \in$ $[1, n-1]$. In $F_{n-1}^{v_{i}, u, v_{i+1}}$, let $f\left(u v_{i}\right)=f\left(u v_{i+1}\right)=x, f\left(u v_{0}\right)=y$, and $f(u)=z$, and the resulting coloring is still denoted by $f$. Evidently, in $F_{n-1}^{v_{i} u, v_{i+1}}, C_{f}(u)=\{x, y, z\}$; meanwhile $C_{f}\left(v_{i}\right)$ and $C_{f}\left(v_{i+1}\right)$ are the same as those in $F_{n-1}$, and $\left|C_{f}\left(v_{0}\right)\right| \geq 5$. So, $f$ a $k$-GVDTC of $F_{n-1}^{v_{i}, u_{i+1}}$.

Case 2. Four different colors $x, y, z, w \in[1, k]$ such that $\{x, y, z, w\} \notin \mathscr{C}_{f}\left(F_{n-1}\right)$. Select an edge $v_{i} v_{i+1}, i \in[1, n-1]$, for which $f\left(v_{i} v_{i+1}\right)=x$. Since $f$ is a $k$-GVDTC of $F_{n-1}$, $C_{f}\left(v_{i}\right) \backslash C_{f}\left(v_{i+1}\right)$ contains at least one element (here we assume $\left.\left|C_{f}\left(v_{i}\right)\right| \geq\left|C_{f}\left(v_{i+1}\right)\right|\right)$, say $c$. Obviously, $c \neq x$, $c \in C_{f}\left(v_{i}\right)$, and $c \notin C_{f}\left(v_{i+1}\right)$ in $F_{n-1}$. We can permutate the colors so that $c \in\{y, x, w\}$ and $f\left(v_{i}\right)=c$ in $F_{n-1}$, say $c=y$. Then, in $F_{n-1}^{v_{i}, u, v_{i+1}}$, erase the color of vertex $v_{i}$ and recolor it by color $x$, and let $f\left(u v_{i}\right)=y, f\left(u v_{i+1}\right)=x$, $f\left(u v_{0}\right)=z$, and $f(u)=w$. Obviously, in $F_{n-1}^{v_{i}, u, v_{i+1}}$, it follows that $C_{f}(u)=\{x, y, z, w\}, C_{f}\left(v_{i}\right)$ and $C_{f}\left(v_{i+1}\right)$ are the same as those in $F_{n-1}$, and $\left|C_{f}\left(v_{0}\right)\right| \geq 5$. So, $f$ a $k$-GVDTC of $F_{n-1}^{v_{i} u, v_{i+1}}$. All of the above show that $F_{n}$ has a $k$-GVDTC.

Theorem 11. Let $W_{n}$ be a wheel graph, $n \geq 2$; then

$$
\chi_{g v t}\left(W_{n}\right)= \begin{cases}3, & n=2,3, \ldots, 6 \\ 4, & n=7,8, \ldots, 14 \\ k, & n \geq 15\end{cases}
$$

where $\left(\begin{array}{c}k-1 \\ 1\end{array}\right)+\left(\begin{array}{c}k-1 \\ 2\end{array}\right)+\left(\begin{array}{c}k-1 \\ 3\end{array}\right)+\left(\begin{array}{c}k-1 \\ 4\end{array}\right)<n \leq\left(\begin{array}{c}k \\ 1\end{array}\right)+\left(\begin{array}{c}k \\ 2\end{array}\right)+\left(\begin{array}{c}k \\ 3\end{array}\right)+\left(\begin{array}{l}k \\ 4\end{array}\right)$.

We omit the proof for Theorem 11, since it is analogous to that of Theorem 10.

Theorem 12. For a complete graph $K_{n}, n \geq 1$, one has

$$
\chi_{\text {gvt }}\left(K_{n}\right)=1+\left\lceil\log _{2} n\right\rceil .
$$

Proof. When $n<10$ the conclusion is easy to show. So we assume $n \geq 10$.

Denote by $V\left(K_{n}\right)=\left\{v_{1}, v_{2}, \ldots, v_{n}\right\}$ and $\{1,2, \ldots, k\}$ the set of $k$ colors. For integer $\ell=\lceil n / 2\rceil$, we construct a $\ell$-GVDTC $f^{\prime}$ of $K_{n}$ as follows: let $f^{\prime}\left(v_{i}\right)=i$ for any $i \in[1, \ell] ; f^{\prime}\left(v_{i} v_{j}\right)=1$ for $i \neq j, i \in[1, \ell]$, and $j \in[1, n] ; f\left(v_{i}\right)=i-\ell+2$ for $i \in[\ell+1, n-2] ; f^{\prime}\left(v_{i} v_{j}\right)=2$ for $i \neq j, i \in[\ell+1, n-2]$, and $j \in[\ell+1, n] ; f^{\prime}\left(v_{n-1} v_{n}\right)=3 ; f^{\prime}\left(v_{n-1}\right)=4 ; f^{\prime}\left(v_{n}\right)=5$. One can readily check that $C_{f}^{\prime}\left(v_{1}\right)=\{1\}, C_{f}^{\prime}\left(v_{i}\right)=\{1, i\}$ for $i=$ $1,2, \ldots, \ell ; C_{f}^{\prime}\left(v_{i}\right)=\{1,2, i-\ell+2\}$ for $i=\ell+1, \ell+2, \ldots, n-2$; $C_{f}^{\prime}\left(v_{n-1}\right)=\{1,2,3,4\}$; and $C_{f}^{\prime}\left(v_{n-1}\right)=\{1,2,3,5\}$. Thus, $f^{\prime}$ is a $\ell$-GVDTC of $K_{n}$, which shows $\chi_{g v t}\left(K_{n}\right) \leq \ell$.

Suppose that $\chi_{\text {gvt }}\left(K_{n}\right)=k$ and $f$ is a $k$-GVDTC of $K_{n}$. Since for any two vertices $v_{i}, v_{j}(i \neq j \in[1, k]), C_{f}\left(v_{i}\right) \cap$ $C_{f}\left(v_{j}\right) \neq \emptyset$, one can see that there is at most one vertex whose total color set contains only one color. If there is a vertex $v$ with $|C(v)|=1$, without loss of generality assume $C(v)=\{1\}$; then the total color set of each vertex contains color 1 , which indicates

$$
n \leq 1+\left(\begin{array}{c}
k-1 \\
1
\end{array}\right)+\left(\begin{array}{c}
k-1 \\
2
\end{array}\right)+\cdots+\left(\begin{array}{l}
k-1 \\
k-1
\end{array}\right)=2^{k-1} .
$$

If there is no vertex whose total color set contains only one color, then for each $v_{i}$ it has $\left|C\left(v_{i}\right)\right| \geq 2$. Since, for any vertex $v_{j} \neq v_{i},\left|C\left(v_{i}\right) \cap C\left(v_{j}\right)\right| \geq 1$, it follows that $C(v)$ and $[1, k] \backslash C(v)$ can not be two total color sets with respect to $f$. This implies $n \leq 2^{k-1}$. So $k \geq 1+\left\lceil\log _{2} n\right\rceil$. 
To prove $k=1+\left\lceil\log _{2} n\right\rceil$, we need to show that $K_{n}$ has a $\left(1+\left\lceil\log _{2} n\right\rceil\right)$-GVDTC. In particular, we show that any $K_{n}$ has a $\left(1+\left\lceil\log _{2} n\right\rceil\right)$-GVDTC such that for each color $c \in[1,1+$ $\left.\left\lceil\log _{2} n\right\rceil\right]$ there is at least one vertex in $K_{n}$ being colored by $c$.

We prove this by induction on $n$. When $n=10,5$ GVDTC is the $f^{\prime}$ defined above for $n=10, \ell=5$, where $C_{f}^{\prime}\left(v_{1}\right)=\{1\}$. Consider the graph $K_{n}-v_{n}$ obtained from $K_{n}$ by deleting vertex $v_{n}$ and its incident edges. Obviously, $K_{n}-v_{n}$ is isomorphic to $K_{n-1}$. By the induction hypothesis, $K_{n-1}$ has a $\left(1+\left\lceil\log _{2} n-1\right\rceil\right)$-GVDTC, say $f$, such that for each color $c \in\left[1,1+\left\lceil\log _{2} n-1\right\rceil\right]$ there is at least one vertex of $K_{n-1}$ being colored by $c$. Since $n \leq 2^{k-1}$, there must be some set $C$ (denoted by $\left.\left\{c_{1}, c_{2}, \ldots, c_{\ell}\right\}\right) \notin \mathscr{C}_{f}\left(K_{n-1}\right)$. We consider the following two cases.

(1) Consider $\left\lceil\log _{2} n-1\right\rceil=\left\lceil\log _{2} n\right\rceil$. By the induction hypothesis, each color $c_{i} \in\left[1,1+\left\lceil\log _{2} n-1\right\rceil\right]$ appears at a vertex. Without loss of generality assume $f\left(v_{i}\right)=$ $c_{i}$ for $i=1,2, \ldots, \ell$. Then, $f$ is extended to a $(1+$ $\left.\left\lceil\log _{2} n-1\right\rceil\right)$-GVDTC of $K_{n}$ via coloring $v_{n}$ by one of the colors in $\left[c_{1}, c_{2}, \ldots, c_{\ell}\right]$; coloring $v_{n} v_{i}$ for $i=$ $1,2, \ldots, \ell$ by $c_{i}$; and coloring $v_{n} v_{j}$ for $j=\ell+1, \ldots, n-1$ by one of the colors in $C_{f}\left(v_{j}\right)\left(v_{j} \in V\left(K_{n-1}\right)\right)$.

(2) Consider $\left\lceil\log _{2} n-1\right\rceil=\left\lceil\log _{2} n\right\rceil-1$. Then on the basis of $f$, we only need to color $v_{n}$ and all of its incident edges in $K_{n}$ by color $1+\left\lceil\log _{2} n-1\right\rceil$.

One can readily check that the resulting coloring of $K_{n}$ in the above two cases is $\left(1+\left\lceil\log _{2} n\right\rceil\right)$-GVDTCs of $K_{n}$ such that each color in $\left[1,1+\left\lceil\log _{2} n\right\rceil\right]$ appears at a vertex of $K_{n}$. Hence, $K_{n}$ has a $\left(1+\left\lceil\log _{2} n\right\rceil\right)-$ GVDTC, and the conclusion holds.

In the following, we present a trivial upper bound of the general vertex-distinguishing total chromatic number of the join graph of two graphs.

Theorem 13. Suppose $G, H$ are two simple graphs and $G \cap H=$ $\emptyset$. Then

$$
\chi_{g v t}(G+H) \leq \chi_{g v t}(G)+\chi_{g v t}(H)
$$

Proof. Let $V(G)=\left\{u_{i} \mid i=1,2, \ldots, m\right\}$ and $V(H)=\left\{v_{i} \mid i=\right.$ $1,2, \ldots, n\}$. Suppose that $f_{1}$ is a $\chi_{g v t}(G)$-GVDTC of $G$ and $f_{2}$ is a $\chi_{\text {gvt }}(H)$-GVDTC of $H$, where the sets of colors of $f_{1}$ and $f_{2}$ are $C_{1}$ and $C_{2}\left(C_{1} \cap C_{2}=\emptyset\right)$, respectively.

Define $f$ as $f\left(u_{i} v_{j}\right)=f_{1}\left(u_{i}\right)\left(\right.$ or $\left.f_{2}\left(v_{j}\right)\right), i=$ $1,2, \ldots, m ; j=1,2, \ldots, n$.

Combining colorings $f_{1}, f_{2}, f$, we can obtain a $\left(\chi_{g v t}(G)+\right.$ $\left.\chi_{g v t}(H)\right)-G V D T C$ of $G+H$.

\section{Remarks}

Based on the above results, we propose two conjectures as follows.

Conjecture 14. Let $G$ be a graph without isolated vertices. Then

$$
\chi_{g v t}(G) \leq\left\lceil\frac{n}{2}\right\rceil
$$

Conjecture 15. Let $G$ be a connected graph on $n$ vertices. Then

$$
\chi_{g v t}(G) \leq 1+\left\lceil\log _{2} n\right\rceil .
$$

Note that if Conjecture 15 is true, then Conjecture 14 is true. On the other hand, if Conjecture 14 is true, then the upper bound cannot be improved. For instance, the graph $G$ contains exactly three $K_{2}$ components. It is easy to show that $\chi_{\text {gvt }}(G)=3$.

In addition, there is a very interesting observation about the general vertex-distinguishing total chromatic number.

Observation 1. Let $H$ be a subgraph of a graph $G$. Then it possibly follows that

$$
\chi_{g v t}(H)>\chi_{g v t}(G) .
$$

As an illustration of this observation, we consider the path $P_{15}$ and the fan graph $F_{14} \cdot P_{15}$ is a subgraph of $F_{14}$, while $\chi_{g v t}\left(P_{15}\right)(=5)>\chi_{g v t}\left(F_{14}\right)(=4)$.

\section{Conflict of Interests}

The authors declare that there is no conflict of interests regarding the publication of this paper.

\section{Acknowledgments}

This work is supported by the National Natural Science Foundation of China (Grant nos. 61127005 and 61309015) and the National Basic Research Program of China (973 Program) (no. 2010CB328103).

\section{References}

[1] J. A. Bondy and U. S. R. Murty, Graph Theory, Springer, 2008.

[2] F. T. Leighton, "A graph coloring algorithm for large scheduling problems," Journal of Research of National Bureau of Standards, vol. 84, no. 6, pp. 489-506, 1979.

[3] D. de Werra and Y. Gay, "Chromatic scheduling and frequency assignment," Discrete Applied Mathematics, vol. 49, no. 1-3, pp. 165-174, 1994.

[4] G. Jonathan and Y. Jay, Graph Theory and Its Applications, CRC press, New York, NY, USA, 1999.

[5] F. Harary, "Conditional colorability in graphs," in Graphs and Applications (Boulder, Colo., 1982), pp. 127-136, John Wiley \& Sons, New York, NY, USA, 1985.

[6] F. Harary and M. Plantholt, "The point-distinguishing chromatic index," in Graphs and Applications, F. Harary and J. S. Maybee, Eds., pp. 147-162, Wiley-Interscience, New York, NY, USA, 1985.

[7] A. C. Burris and R. H. Schelp, "Vertex-distinguishing proper edge-colorings," Journal of Graph Theory, vol. 26, no. 2, pp. 7382, 1997.

[8] Z. Zhang, L. Z. Liu, and J. F. Wang, "Adjacent strong edge coloring of graphs," Applied Mathematics Letters, vol. 15, no. 5, pp. 623-626, 2002.

[9] E. Györi, M. Horñák, C. Palmer, and M. Woźniak, "General neighbour-distinguishing index of a graph," Discrete Mathematics, vol. 308, no. 5-6, pp. 827-831, 2008. 
[10] M. Behzad, Graphs and Their Chromatic Numbers, Michigan State University, 1965.

[11] V. G. Vizing, "Some unsolved problems in Graph Theory," Russian Mathematical Surveys, vol. 23, pp. 125-141, 1968.

[12] Z. Zhang, X. E. Chen, J. Li, B. Yao, X. Lu, and J. Wang, "On adjacent-vertex-distinguishing total coloring of graphs," Science in China. Series A. Mathematics, vol. 48, no. 3, pp. 289-299, 2005.

[13] X. Chen, "On the adjacent vertex distinguishing total coloring numbers of graphs with $\Delta=3$," Discrete Mathematics, vol. 308, no. 17, pp. 4003-4007, 2008.

[14] H. Wang, "On the adjacent vertex-distinguishing total chromatic numbers of the graphs with $\Delta=3$," Journal of Combinatorial Optimization, vol. 14, no. 1, pp. 87-109, 2007.

[15] J. Hulgan, "Concise proofs for adjacent vertex-distinguishing total colorings," Discrete Mathematics, vol. 309, no. 8, pp. 25482550, 2009.

[16] D. Huang, W. Wang, and C. Yan, "A note on the adjacent vertex distinguishing total chromatic number of graphs," Discrete Mathematics, vol. 312, no. 24, pp. 3544-3546, 2012.

[17] M. Chen and X. Guo, "Adjacent vertex-distinguishing edge and total chromatic numbers of hypercubes," Information Processing Letters, vol. 109, no. 12, pp. 599-602, 2009.

[18] Y. Wang and W. Wang, "Adjacent vertex distinguishing total colorings of outerplanar graphs," Journal of Combinatorial Optimization, vol. 19, no. 2, pp. 123-133, 2010.

[19] V. Pedrotti and C. P. de Mello, "Adjacent-vertex-distinguishing total coloring of indifference graphs," Matemática Contemporânea, vol. 39, pp. 101-110, 2010.

[20] W. Wang and D. Huang, "The adjacent vertex distinguishing total coloring of planar graphs," Journal of Combinatorial Optimization, vol. 27, no. 2, pp. 379-396, 2014.

[21] X. Chen and Z. Zhang, "AVDTC numbers of generalized Halin graphs with maximum degree at least 6," Acta Mathematicae Applicatae Sinica, vol. 24, no. 1, pp. 55-58, 2008.

[22] D. Huang, W. Wang, and C. Yan, "A note on the adjacent vertex distinguishing total chromatic number of graphs," Discrete Mathematics, vol. 312, no. 24, pp. 3544-3546, 2012.

[23] A. Papaioannou and C. Raftopoulou, "On the AVDTC of 4regular graphs," Discrete Mathematics, vol. 330, pp. 20-40, 2014.

[24] Z. Zhang, P. X. Qiu, B. G. Xu, J. Li, X. Chen, and B. Yao, "Vertexdistinguishing total coloring of graphs," Ars Combinatoria, vol. 87, pp. 33-45, 2008. 


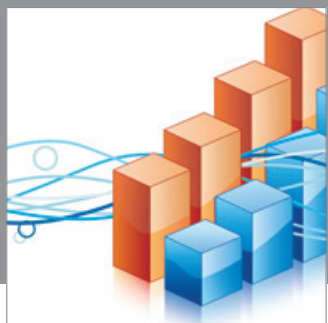

Advances in

Operations Research

mansans

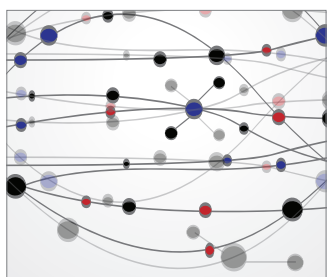

The Scientific World Journal
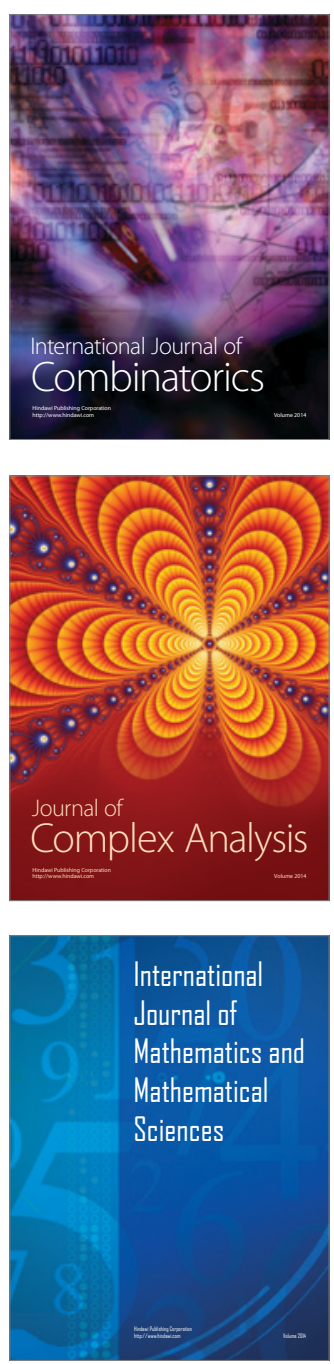
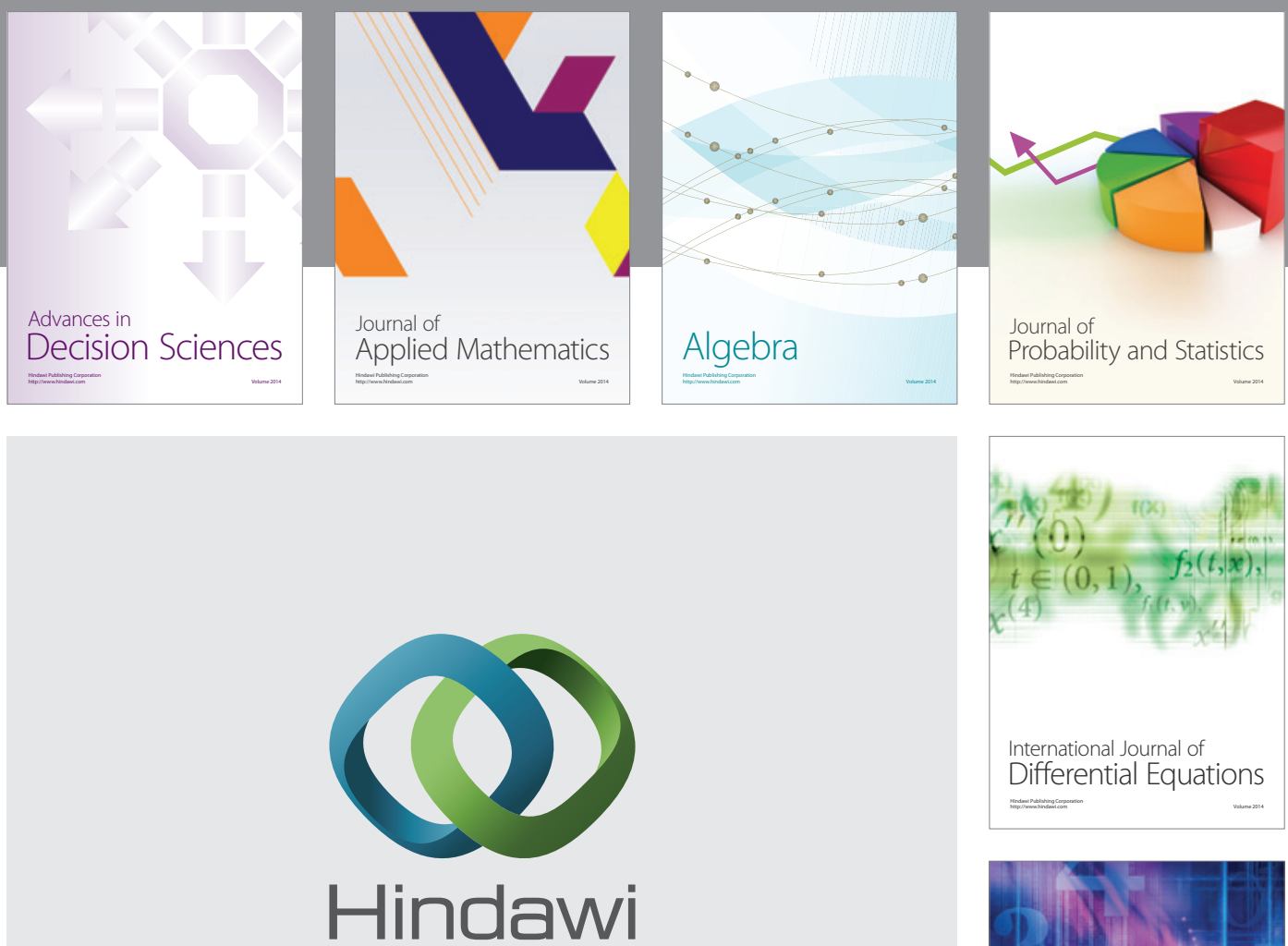

Submit your manuscripts at http://www.hindawi.com
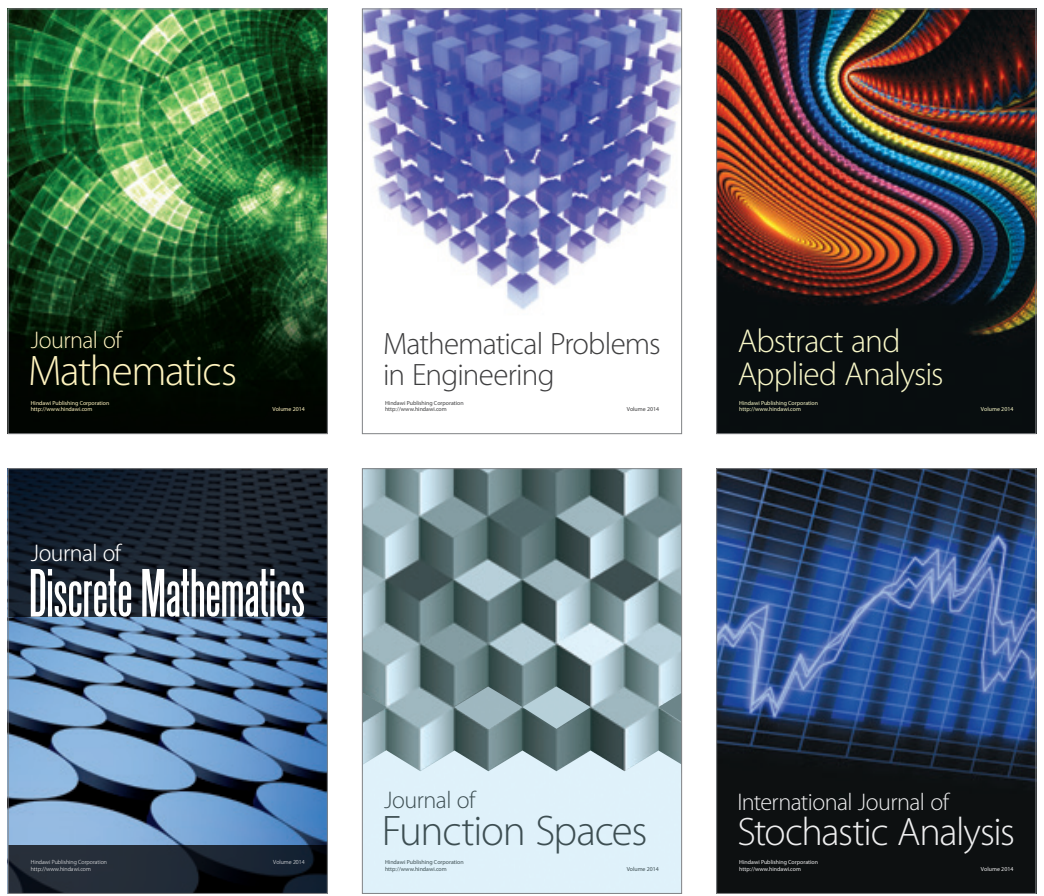

Journal of

Function Spaces

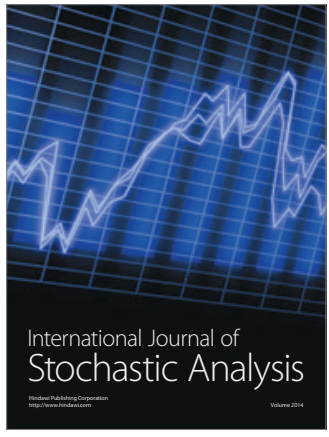

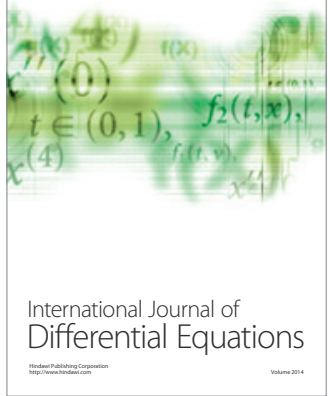
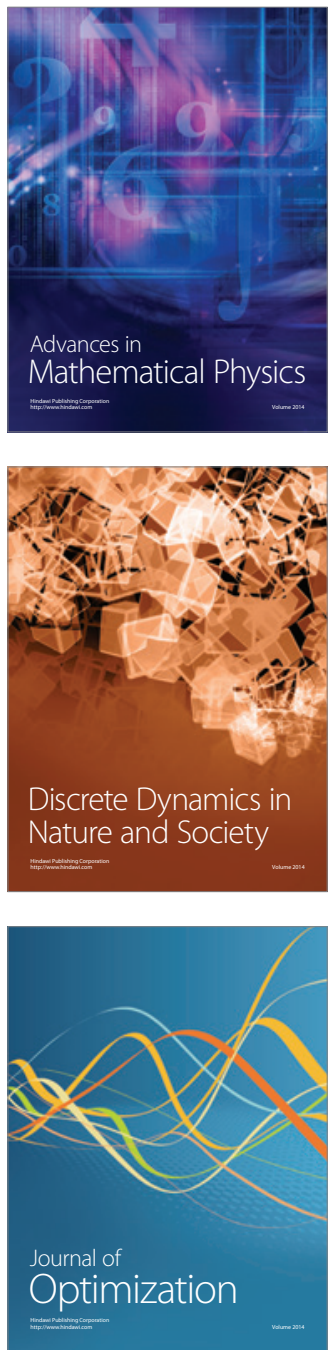